\title{
Plasmon-Enhanced Non-Radiative Energy Transfer in a Hybrid Quantum Well Structure
}

\author{
L.J. Higgins ${ }^{1}$, V.D. Karanikolas ${ }^{1}$, G.P. Murphy ${ }^{1}$, X. Zhang ${ }^{1}$, C.A. Marocico ${ }^{1}$, P.J. Parbrook ${ }^{2}$, \\ and A.L. Bradley ${ }^{*}$ \\ ${ }^{1}$ Department of Physics and CRANN, Trinity College Dublin, College Green, Dublin 2, Ireland \\ ${ }^{2}$ Tyndall National Institute and School of Engineering, University College Cork, \\ Lee Maltings, Prospect Row, Cork, Ireland \\ e-mail:bradlel@tcd.ie
}

\begin{abstract}
Three different silver metal nanobox arrays are used to demonstrate plasmon-enhanced non-radiative energy transfer from an InGaN/GaN quantum well to a $\sim 80 \mathrm{~nm}$ thick layer of $\mathrm{CdSe} / \mathrm{ZnS}$ quantum dots embedded in PMMA. These arrays of varying periodicity are fabricated using helium-ion lithography. Whilst no direct signatures of non-radiative energy transfer are detected in the absence of the Ag nanobox arrays, we observe plasmon-enhanced non-radiative energy transfer efficiencies of up to $30 \%$ from the quantum well to the quantum dots. We show that by tuning the Ag nanobox array periodicity the acceptor QD emission can be optimized to benefit from both non-radiative energy transfer and direct plasmonic enhancement of the QD emission. QD emission enhancements of up to $71 \%$ were observed and QD lifetime enhancements of $\sim 50 \%$ are also reported.

Keywords: plasmonics, non-radiative energy transfer, helium-ion lithography, quantum well, quantum dot, arrayed geometries.
\end{abstract}

\section{INTRODUCTION}

In this work, we investigate the use of three different silver nanobox arrays to facilitate localised surface plasmon - enhanced non-radiative energy transfer (LSP-NRET) in a hybrid quantum well (QW) - quantum dot (QD) structure. The Ag nanobox arrays are fabricated on top of a $3 \mathrm{~nm}$ thick capping layer of a single InGaN/GaN QW using helium-ion lithography (HIL). The QW acts as the donor for the energy transfer system. The acceptors are CdSe/ZnS QDs embedded in a $\sim 80 \mathrm{~nm}$ thick PMMA layer. HIL allows for high resolution fabrication of periodic nanostructures. This level of control over the shape, size and periodicity of the nanostructures enables sensitive tailoring of the surface plasmon resonance (SPR) and the local electromagnetic field. In this work the individual shape and size of the nanoboxes are fixed but the periodicity within the array is varied. The nanobox arrays can separately influence the QW and QD emission as well as the LSP-NRET between them. LSP-coupled emission and LSP-NRET are sensitive to SPR properties, such as the spectral overlap with emission and/or absorption features and the local field strength [1-2]. We use time-resolved photoluminescence (TRPL) and PL spectral measurements to investigate the impact of the Ag nanoboxes on both the LSP-NRET and the total emission from the full hybrid system.

\section{RESULTS AND DISCUSSION}

A schematic of the full hybrid structure is shown in Fig. 1(a). The full structure consists of an InGaN/GaN QW with lithographically defined silver nanobox arrays fabricated by HIL built directly on top, and subsequently covered with a layer CdSe/ZnS QDs embedded in PMMA, which is deposited by the spin-coating technique. Three different nanobox arrays are investigated in this work, labelled Box 1, Box 2 and Box 3. They consist of Ag nanoboxes with a lateral dimension of $100 \mathrm{~nm}$, height of $35 \mathrm{~nm}$ and separations of $100 \mathrm{~nm}, 130 \mathrm{~nm}$ and $160 \mathrm{~nm}$, respectively. A S.E.M. image of the Box 1 array is shown in Fig. 1(b). It can be seen that the large array of boxes is very uniform and that the aspect ratio of the individual boxes is well maintained.

Time-resolved photoluminescence (TRPL) measurements were taken to investigate the presence of NRET from the QW to the Ag nanobox arrays and directly to the QDs. The TRPL decay curves are fit with a biexponential and the average PL lifetime is given by Eqn.1,

$$
\tau_{\text {avg }}=\frac{I_{1} * \tau_{1}^{2}+I_{2} * \tau_{2}^{2}}{I_{1} * \tau_{1}+I_{2} * \tau_{2}},
$$

where $I_{1}$ and $I_{2}$ are the intensity amplitudes for the exponential decays with lifetimes $\tau_{1}$ and $\tau_{2}$, respectively. 


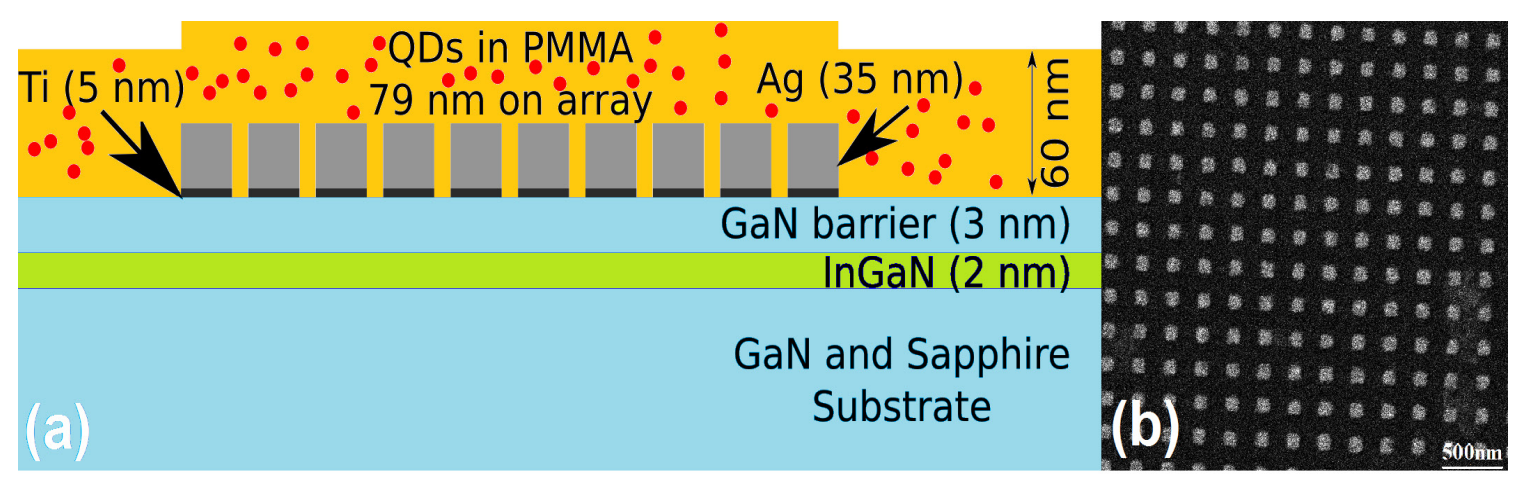

Figure 1: (a) Schematic of full hybrid structure; (b) S.E.M. image of Box 1 array.

The nanobox arrays can separately influence the QW and QD emission as well as the LSP-NRET between them. The spectral overlap with emission and/or absorption features can strongly influence both processes. Figure 2(a) shows the emission spectra of the QW and the QDs, the absorption spectrum of the QDs and the extinction spectra for the three nanobox arrays. The QW TRPL decay curves for the QW only, the QW with Box 1 array fabricated on top of the capping layer and for the full structure with the QD layer, are shown in Fig. 2(b). The QW and QW-nanobox array structures are also coated with a $\sim 80 \mathrm{~nm}$ thick PMMA layer to allow for direct comparison.

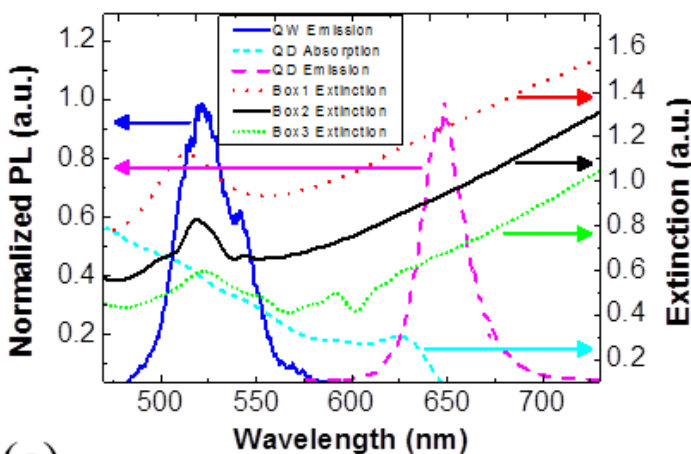

(a)

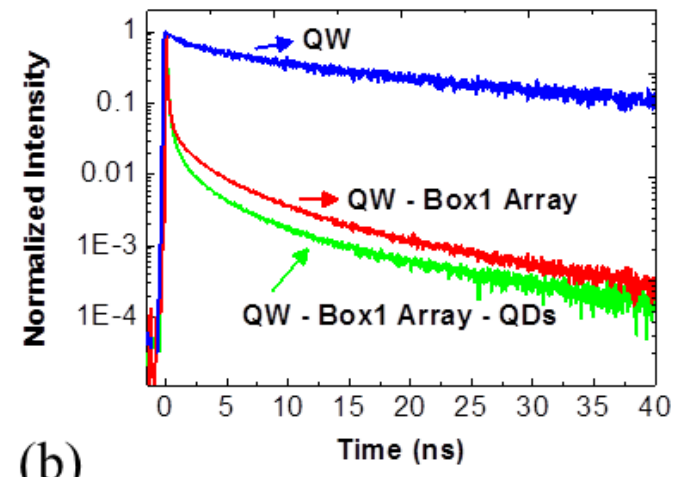

(b)

Figure 2: (a) The normalized emission spectra of the $Q W$ and $Q D s$ along with the absorption spectrum of the $Q D s$ and the theoretically modelled extinction spectra of the three Ag nanobox arrays; (b) Normalized PL decay curves for the $Q W$ by itself (blue line), $Q W$ on the Box 1 array (red line) and $Q W$ on Box 1 array after deposition of the QD layer (green line).

The reduction of the QW lifetime and PL emission when measured on the Box1 array is a signature of quenching of the QW emission by the nanobox array. The quenching efficiency can be quantified by Eqn. 2 [3].

$$
E_{Q}=1-\frac{\tau_{Q W-N B}}{\tau_{Q W}}
$$

The further reduction in the QW lifetime after the deposition of the QDs is a signature of LSP-NRET from the QW to the QDs. The LSP-NRET efficiency is given by Eqn.3 [3].

$$
E_{N R E T}=1-\frac{\tau_{Q W-N B-Q D}}{\tau_{Q W-N B}}
$$

By using both Eqns. (2) and (3), the quenching and LSP-NRET efficiencies are calculated for all three nanobox arrays, shown in Fig. 3(a). In all cases it can be seen that the quenching efficiency is approximately $90 \%$ and that the plasmon-enhanced energy transfer efficiencies vary from $\sim 25 \%-30 \%$. Therefore, the three nanobox structures appear to facilitate similar levels of NRET from the QW to the QDs. It should be noted at this point that there is no evidence of NRET from the QW to the QD layer in the absence of the nanobox arrays, signified by no measureable change in the donor QW lifetime. 


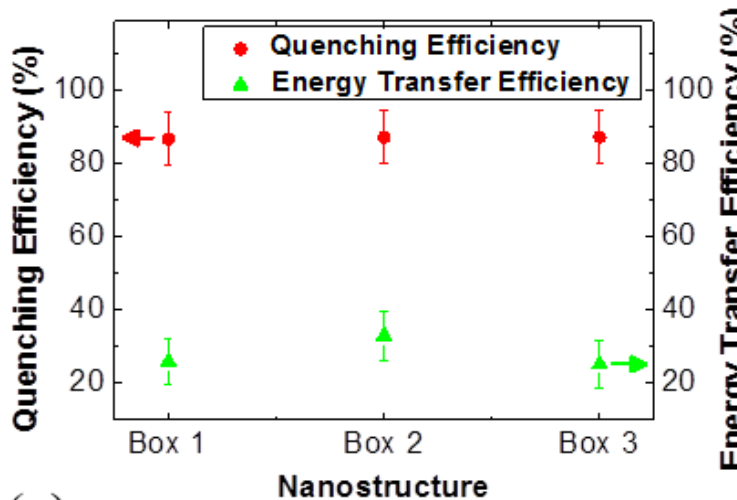

(a)

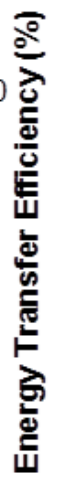

(b)

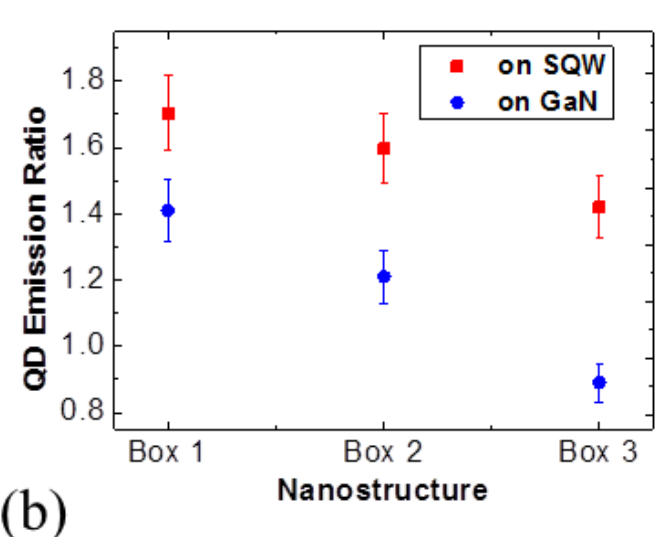

Nanostructure

Figure 3: (a) Quenching efficiencies and plasmon-enhanced energy transfer efficiencies as a function of nanobox array; (b) $Q D$ emission ratio on both $Q W$ and bulk GaN substrates as a function of nanobox array.

Next we consider the impact of changing the periodicity of the nanobox array on the total emission from the hybrid system. We have already seen that there has been strong direct quenching of the QW emission. To investigate the impact of the different nanobox arrays on the QDs, the QD emission ratio (QD emission on the structure/QD emission off the structure) on both the SQW and bulk GaN substrates is plotted in Fig. 3(b). The QD emission ratio on the GaN substrate reveals the intrinsic interaction between the QDs and the nanobox arrays. The QD emission is quenched on Box3 and enhanced on Box1 and Box2 arrays. The QD emission on the nanobox arrays on the SQW substrate is further increased in all cases. QD emission enhancement varies from $43 \%$ for Box3 to $71 \%$ for Box1. LSP-NRET has facilitated a significant increase in the QD emission, and furthermore, it is also possible to optimize the QD emission benefitting from both LSP-NRET and direct enhancement of the QD emission by tuning the array periodicity.

We can also consider the impact of the nanobox arrays on the PL lifetime of the QDs. Figure 4(a) shows the QD PL decays on and off Box1 array fabricated on a bulk GaN substrate. This allows us to investigate the interaction between the QDs and the Ag nanoboxes with the same excitation source but in the absence of the donor QW. A $(29 \% \pm 3 \%)$ reduction in the QD average PL lifetime is observed on the array relative to off the array. Furthermore, Fig. 4(b) shows the QD PL decays both on and off Box1 array on the QW substrate. In this case the QDs on the array show an $(6 \% \pm 2 \%)$ increase of the PL lifetime relative to QDs off the array. Therefore, the influence of the plasmon-enhanced energy transfer is sufficiently strong that the QD PL lifetime not only recovers from the reduction on the array but is even further increased. The decay is measured over $80 \mathrm{~ns}$ but the first $10 \mathrm{~ns}$ is given in Fig. 4 to more clearly show the change in the PL decay.
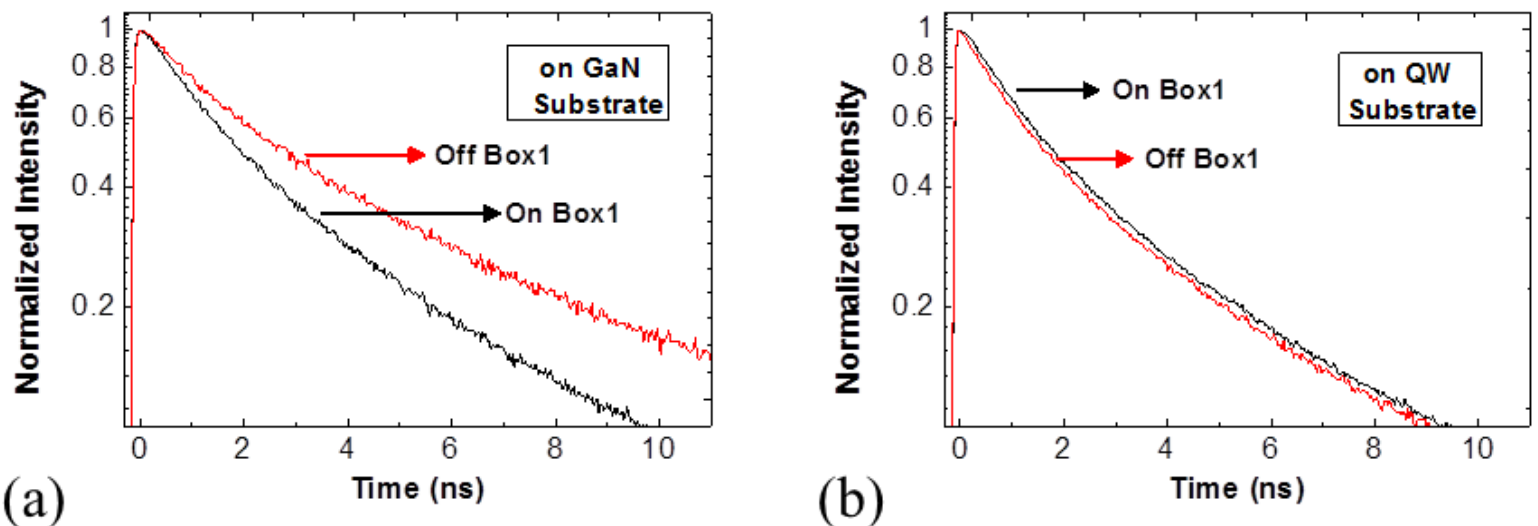

Figure 4: (a) The QD PL decay on and off the Ag nanobox array on a GaN bulk substrate; (b) $Q D P L$ decay on and off of Ag nanobox array on the $Q W$ sample.

We can now consider the level of QD lifetime enhancement (lifetime ratio on/off array on QW substrate divided by the lifetime ratio on/off array on $\mathrm{GaN}$ substrate) for all three nanobox arrays. Figure 5 shows both the LSP-NRET efficiency and the QD lifetime enhancement for the three arrays. We can see that a QD lifetime enhancement of $\sim 50 \%$ is observed for all three arrays and that the enhancement is relatively insensitive to the changes in periodicity. This is consistent with the insensitivity to periodicity changes seen in the LSP-NRET efficiencies. 


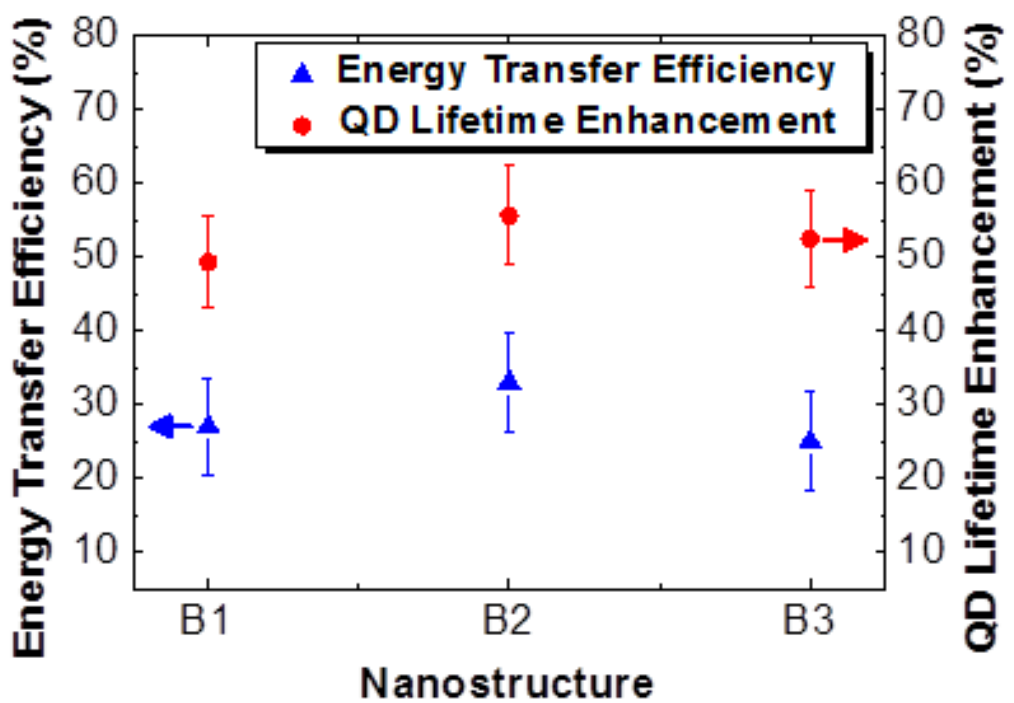

Figure 5. Energy transfer efficiencies (blue triangles) and QD lifetime enhancements (red circles) as a function of nanostructure.

\section{CONCLUSIONS}

In conclusion, we have demonstrated LSP-NRET from an InGaN/GaN QW to an $\sim 80 \mathrm{~nm}$ thick layer of QDs embedded in PMMA using Ag nanobox arrays fabricated by HIL. Three nanobox arrays geometries with different periodicities were fabricated to study their impact on LSP-NRET and overall QD emission. There is no measureable direct NRET from the QW to the QD layer in the absence of the nanobox arrays. We find that the nanoboxes substantially increase the LSP-NRET efficiency to $\sim 30 \%$ despite strong direct quenching of the QW emission. The LSP-NRET did not show strong sensitivity to the periodicity. However, we did observe that QD emission enhancements could be optimized by tuning the periodicity. The emission enhancement had contributions from both direct LSP-enhanced emission and LSP-NRET. QD PL emission enhancements of up to $71 \%$ were observed. Furthermore, both the direct LSP-enhanced emission and LSP-NRET processes were observed to impact the QD lifetimes. These lifetimes were investigated by fabricating the nanobox arrays on both bulk GaN and SQW substrates. QD lifetime enhancement did not show strong sensitivity to the periodicity either, which was consistent with the insensitivity seen for the LSP-NRET efficiency. QD lifetime enhancements of $\sim 50 \%$ were observed for all three arrays. Plasmon-coupled non-radiative energy transfer may alleviate some of the constraints on the use of non-radiative pumping for down-conversion based LEDs, such as the need for very thin QD layers or etching of the QW structures.

\section{ACKNOWLEDGEMENTS}

This work was supported by Science Foundation Ireland (SFI) under grant number 10/IN.1/12975, the National Access Programme Grant under grant number NAP 338 and the Irish Research Council under grant number RS/2011/287. PJP acknowledges a SFI Engineering Professorship (SFI/07/EN/E001A). We also would like to acknowledge the Advanced Microscopy Laboratory (AML) for use of the helium-ion microscope.

\section{REFERENCES}

[1] T. Forster, "Zwischenmolekulare Energiewanderung und Fluoreszenz," Annalen Der Physik 437(1-2), 55-75, (1948).

[2] V.M. Agranovich, G.C. La Rocca, and F. Bassani, "Efficient electronic energy transfer from a semiconductor quantum well to an organic material," JETP Lett. 66, 748 (1997).

[3] L.J. Higgins, V.D. Karanikolas, C.A. Marocico, A.P. Bell, T.C. Sadler, P.J. Parbrook, and A.L. Bradley, "Carrier density dependence of plasmon-enhanced nonradiative energy transfer in a hybrid quantum wellquantum dot structure," Optics Express 23(2), 1377-1387 (2015). 